\title{
Modélisation numérique de transport de vases et de la salinité dans un complexe lagunaire
}

\author{
Z.X. Fang, M. Cavailles, J. Astier \\ Ingénieurs de $B R L$ ingénierie, Nîmes
}

\begin{abstract}
This paper presents the numerical models designed and used to the study of BagesSigean lagoon (on French Mediterranean coast).

Two models are concerned: a two dimensional depth averaged finite element model for hydrodynamics and a conceptual model for cohesive sediments transport (clay bed) and salinity transfert. The second model is based on the classic convectiondiffusion equation; it uses the water surface elevation and velocities generated by the hydrodynamic computations. The clay deposition and bed erosion rates are calculated with the formulations of Krone and Patheriades respectively. The study case is representative of most of the Mediterranean coastal estuaries where the bed is composed of clay in the marority and the water flow is shallow.
\end{abstract}

\section{Introduction}

L'étang de Bages-Sigean est situé dans le département de l'Aude, et s'étend du sud au nord, de Port-la-Nouvellejusqu'au sud de Narbonne, sur une superficie d'environ $37 \mathrm{~km}^{2}$ (voir Carte 1). Ce milieu s'étire sur plus de $14 \mathrm{~km}$ dans sa plus grande longueur et sur $6 \mathrm{~km}$ dans sa plus grande largeur.

L'étang communique avec la mer par le chenal de Port-la-Nouvelle où se sont développées des infrastructures portuaires (pêches, transport d'hydrocarbures et de produits agro-alimentaires). Afin de limiter l'envasement du port par les sédiments en provenance de l'étang (essentiellement des vases), un barrage à vannes a été implanté en têtte du chenal. Plusieurs études récentes menées par le laboratoire ARAGO, BRL $i$ et CEPRALMAR ont montré que la tendance à l'eutrophisation du milieu lagunaire et la baisse des captures par les pêcheurs étaient imputables en partie à l'existence de cet ouvrage.

L'enlèvement de cet ouvrage entrainera certainement des modifications importantes des échanges hydrauliques et du transport sédimentaire dans le chenal de Port-laNouvelle. Avant d'engager ce projet, il y a donc lieu de vérifier les impacts, notamment sur l'envasement du chenal et les coûts de dragage qui sont aujourd'hui déjà très élevés. 
L'étude menée par BRLi sur les impacts de la modification de l'ouvrage concerne en effet plusieurs domaines : modélisation, génie civil, hydrobiologie etc. Cet article présente en particulier l'aspect "modélisation" de l'étude. Deux modèles découplés ont été construits dans le cadre de l'étude : modèle hydrodynamique et modèle de convection - diffusion pour le transport de vases et le transfert de salinité. Ces modèles ont été calés à partir des données des différentes sources: les niveaux d'eau de la mer et de l'étang (SMNLR), la salinités dans l'étang (AME), les vents et les précipitations (Météo France), les apports du bassin versant (DIREN), etc.

Le modèle hydrodynamique est bidimensionnel plan en éléments finis; les simulations ont été effectuées au pas de temps de $12 \mathrm{~h}$ sur plusieurs années; les caractéristiques obtenues sont les hauteurs d'eau, le champ de vitesse et les débits de courants. Ce modèle est fondamental du fait que :

- il permet d'évaluer directement l'impact du projet d'aménagement de l'ouvrage sur les échanges hyưrauliques entre l'étang et la mer,

- il fournit les données hydrodynamiquesnécessaires à la modélisation du transport de vases et de la salinité, à savoir les hauteurs d'eau et les vitesses de courants.

La modélisation du transport de vases consiste à résoudre l'équation classique de convection - diffusion avec la prise en compte des particularités liées aux phénomènes de dépôt - érosion des sédiments cohésifs. Le modèle de transport est également bidimensionnel horizontal en utilisant des variables intégrées sur la verticale. La réaction des particules solides sur l'écoulement est négligée. En outre le modèle suppose un découplage entre l'hydraulique et la sédimentologie: ceci signifie que l'évolution des fonds est lente devant l'échelle hydraulique, en d'autres termes les calculs hydrauliques ne seront réactualisés que lorsque l'évolution des fonds devient significative.

Ces hypothèses sont relativement vérifiées pour le milieu étudié caractérisé par des fines particules (donc faible vitesse de chute), des faibles profondeurs et des concentration modérées. L'objectif principal de la modélisation du transport de vases est d'évaluer les volumes de sédiments déposés dans le chenal maritime en provenance de l'étang en fonction des différentes hypothèses d'aménagement. Le modèle de transport conçu pour l'étude sédimentologique a été adapté à la simulation de la salinité dans les différentes parties de l'étang. 
Le complexe lagunaire de Bages-Sigean est assez représentatif des étangs littoraux Méditerranéens du point de vue des caractéristiques des sédiments et de la configuration topographique. Les données hydrologiques, météorologiques et sédimentologiques, observées sur des périodes communes, nous ont permis de caler et mettre au point le modèle. Ce modèle constitue indéniablement une avancée dans l'élaboration d'outils d'aide à la décision car il apporte des connaissances sur l'hydrodynamisme, la sédimentologie et la qualité des eaux de lagune. Les résultats obtenus permettront ainsi de mieux comprendre le fonctionnement de l'écosystème lagunaire et surtout de quantifier les impacts d'aménagements qui visent à modifier les mouvements des masses d'eau à l'intérieur des lagunes et les échanges mer étang, (renouvellement des eaux, mise en suspension et dépôt de vase, vitesse de courant, etc.).

Remerciements : Nous remercions vivement M. BOUGIS, ingénieur indépendant, et Mademoiselle MONTALIEU, ingénieur ISITV pour leur contribution.

\section{Méthodologie}

\subsection{Modélisation hydrodynamique}

Le secteur modélisé comprend l'étang, le chenal maritime et une zone en débouché de la mer. Le niveau de la mer à Port-la-Nouvelle a été mesuré par SMNLR durant 1993-1996, il constitue une condition aux limite du modèle. Les autres conditions aux limites sont: apports propres de l'eau douce (apports du bassin versant + impluvium sur la surface de l'étang - évaporation dans l'étang) et les vents.

L'équation de base est celle de Barré de St Venant. L'hypothèse de la vitesse intégrée sur la hauteur est acceptable dans la mesure où les profondeurs sont très faibles ( $1.3 \mathrm{~m}$ en moyenne).

Le vent agit sur les eaux de surface et a un effet d'entrainement dynamique sur la masse d'eau, il joue en effet un rôle déterminant dans le processus hydrodynamique du système modélisé, où la Tramontane ou les vents marins soufflent quasiment en permanence.

Deux types de mouvements sont provoqués par le vent pour les particules d'eau :

- déplacement dans les courants,

- rotation due aux vagues ou clapots. 
Le deuxième type de mouvement est négligé dans la modélisation hydrodynamique mais pris en compte dans le modèle du transport.

La contrainte de frottement du vent sur la surface de l'eau est proportionnelle à $\mathrm{V}^{2}$ (vitesse de vent) d'après la formule de WU. A titre indicatif un vent de $12 \mathrm{~m} / \mathrm{s}$ peut provoquer un débit maximum d'écoulement dans le chenal de $30 \mathrm{~m} / \mathrm{s}$ pour un niveau initialement équilibré entre l'étang et la mer; ce débit atteindra plus que $100 \mathrm{~m}^{3} / \mathrm{s}$ si l'on considère la décote de la mer entraînée par le même vent. A noter que le chenal maritime de Port-la-Nouvelle a une largeur de l'ordre de $100 \mathrm{~m}$ et une profondeur de 4 à $9 \mathrm{~m}$.

Le modèle hydrodynamique a pu être calé grâce aux données limnigraphiques sur deux points dans l'étang: on a comparé en effet les cotes calculées à celles observées mois par mois. Un exemple des graphiques utilisés pour le calage et une carte illustrant le champ de vitesses sont fournis ci-après.

\subsection{Modélisation du transport}

Les trois termes de l'équation convecto-diffusivesont :

- convection due aux courants d'eau,

- diffusion turbulente,

- termes source: remise en suspension, dépôt, biodégradation, réaction chimique, ...

Les sédiments vaseux cohésifs ont ses particularités, on peut citer la description de BONNEFILLE :

- le transport et le dépôt ne dépendent pas directement de la granulométrie des particules mais plutôt de la taille des agrégats formés par le phénomène de floculation. En eau calme, les vitesses de chute des particules de vases $(0,1$ à $40 \mathrm{~mm}$ ) sont très lentes $\left(1 \mathrm{~mm}\right.$ en 10 à $10^{6} \mathrm{~s}$ ). En revanche en milieux aqueux contenant des sels dissous, comme dans l'étang de Bages-Sigean, les particules se rassemblent en flocons de 0,5 à $1,5 \mathrm{~mm}$, dont les vitesses de chute sont 10 à 100000 fois plus grandes que celles des particules individuelles, ce phénomène appelé floculation, est d'autant plus poussé que la concentration en particules solides, la salinité et la température sont élevées.

- la remise en suspension est faite par arrachement de bouffées de vase: le paramètre de base pour quantifier la vitesse d'arrachement est la rigidité des différentes couches de sédiments entassés au fond, qui elle-même est liée à la concentration(de l'ordre de 100 à $300 \mathrm{~g} / \mathrm{l}$ pour la première couche). 
Le "transport" et la "dispersion" des particules solides dans la masse d'eau, dont les mouvements ont été déterminés par un modèle hydraulique séparé, consistent simplement à résoudre l'équation, il reste ici à évaluer les termes source, c'est à dire les échanges entre l'écoulement fluide et le fond. Les principales lois utilisées dans le modèle de Bages-Sigean sont les suivantes :

- formulation de Partheniades pour la remise en suspension, qui suppose que le flux d'érosion dépend linéairement de l'excès de contrainte de cisaillement sur le fond par rapport à la contrainte critique de début d'entraînement. La contrainte critique elle-même dépend des caractéristiques du tassement (âge, concentration, épaisseur, etc.),

- formule de Krone pour le flux de dépôt, qui est relié à la vitesse de chute des particules (en effet ce sont des agrégats de vases dans l'eau salée) et à la condition de mouvement d'eau caractérisée par la contrainte de cisaillement au fond,

- formule de Bijker pour la contrainte de cisaillement au fond en fonction de la vitesse orbitale des particules d'eau due aux vagues (ou clapots) et de la vitesse de courant,

- formulation proposée dans SHORE PROTECTION MANUAL pour les caractéristiques des vagues (hauteur, période, longueur etc.), adaptée aux faibles profondeurs.

Les calculs des différentes étapes nous ont permis de comprendre plusieurs phénomènes :

- dans le mécanisme de la remise en suspension, les vagues sont le responsable principal. En effet la vitesse orbitale de l'eau est d'un ordre supérieur à celle de courant. A titre indicatif, la vitesse de courant est de l'ordre de $0.02 \mathrm{~m} / \mathrm{s}$ et la vitesse orbitale de $0.2 \mathrm{~m} / \mathrm{s}$;

- de ce fait la remise en suspension dans des zones relativement profondes tel que le chenal maritime ne peut pas être provoquée par le vent, car l'agitation au fond est faible ;

- du fait que le volume des sédiments peut s'accumuler dans l'eau, la concentration dépend de la séquence des vents: il n'y a pas de corrélation simple entre le vent et la turbidité ;

- la sédimentation ne se réalise que lorsque la force de vent est inférieure au minimum nécessaire à la remise en suspension, autrement dit il n'y a pas d'érosion et sédimentation simultanées. Dans cette condition le pas de temps de calcul doit être suffisamment petit pour pouvoir représenter correctement l'alternatifdes épisodes de vitesses de vent fortes et faibles. 
Le modèle a été calé sur quelques observations de la concentration (CATOLLIOTI, MILLET et GRILLAS etc.) et globalement à partir des données de dragage fournis par SMNLR. Le même modèle a été utilisé pour étudier le transfert de la salinité, en affectant des valeurs nulles aux termes source de l'équation convecto - diffusive. Un calage du modèle de transport a été également réalisé à partir des observations de la salinité dans les différentes parties de l'étang.

\section{Résultats obtenus}

L'hypothèse principale d'aménagement consiste en effet à démolir le barrage à vannes sur le chenal, dans ce cas la section mouillée minimum passerait de $54 \mathrm{~m}^{2}$ à l'état actuel à $134 \mathrm{~m}^{2}$ (pont de $\mathrm{SNCF}$ ). Les résultats de la modélisation sont suivants :

- les volumes d'eau échangés entre la mer et l'étang seront augmentés de $28 \%$,

- l'envasement du port sera augmenté de 15 à $25 \%$, soit un coût supplémentaire de 1 à 1.75 millions de francs par an,

- l'impact sur la salinité dans le nord de l'étang sera faible (voir graphique ciaprès),

- la vitesse maximale du courant dans le chenal (près de $2 \mathrm{~m} / \mathrm{s}$ au niveau de l'ouvrage) sera réduite de plus de moitié,

- l'hydrodynamisme de l'étang (courants d'eau) ne sera pas modifié, mais le mouvement des sédiments sera influencé.

Ces résultats ont permis d'évaluer ensuite les différents impacts économique, hydrobiologique et environnemental. Outre l'hypothèse de base plusieurs autres scénarios d'aménagement ont été examinés.

\section{Conclusion}

La modélisation numérique constitue incontestablementun outil précieux d'aide à la décision. Aujourd'hui grâce aux progrès informatiques des résultats fiables peuvent être obtenus rapidement avec un coût très limité.

Dans le cas de Bages-Sigean les résultats sont très encourageants bien que le système modélisé soit complexe: ils ont permis de bien appréhender le fonctionnement hydrodynamique et sédimentologique de l'étang et de comprendre certains phénomènes liés aux systèmes lagunaires mal connus jusqu'à présent.

Les modèles, conçus dans le cadre de l'étude pour répondre à une demande précise, peuvent en effet adaptés facilement à d'autres systèmes similaires et éventuellement à d'autres types de matières en suspension tels que des polluants. 
Certains problèmes mal résolus actuellement nécessiteront encore des investigations: on peut noter les paramètres suivants dont la détermination est aujourd'hui encore très empirique :

- rigidité des couches sédimentaires aux fonds liées à la condition de tassement,

- coefficient de diffusion turbulente,

- vitesse de chute des particules (flocons) en eau calme en fonction de la salinité et des caractéristiques granulométriques des sédiments, ...

\section{BIBLIOGRAPHIE}

ARIATHURAI R., MACARTHUR R. C. \& KRONE R. B. 1977, "Mathematical model of estuarial sediment transport", Technical report D-77-12, US Army Engineer Waterways Experiment Station.

BONNEFILLE R. 1992, "Cours d'hydraulique maritime", 3 ème édition, Masson.

Brigham Young University 1994, RMA2/RMA4 user's manual.

CATALIOTTI-VALDINA D. 1978, "Modalités et bilan de la sédimentation pélitique dans un milieu margino-littoral". Le complexe lagunaire de Bages-SigeanPort la Nouvelle. Thèse de spécialité, Université de Toulouse.

MILLET B. \& GRILlAS P. 1995, "Modélisation numérique de la remise en suspension du sédiment dans l'étang de Vaccarès", non publié.

ROIG L. C. et al. 1996, SED2D-WES user's manual, US Army Engineer Waterways Experiment Station.

US Army Corps of Engineers 1984, "Shore protection manual", $2^{\text {ème }}$ édition.

Figure 1

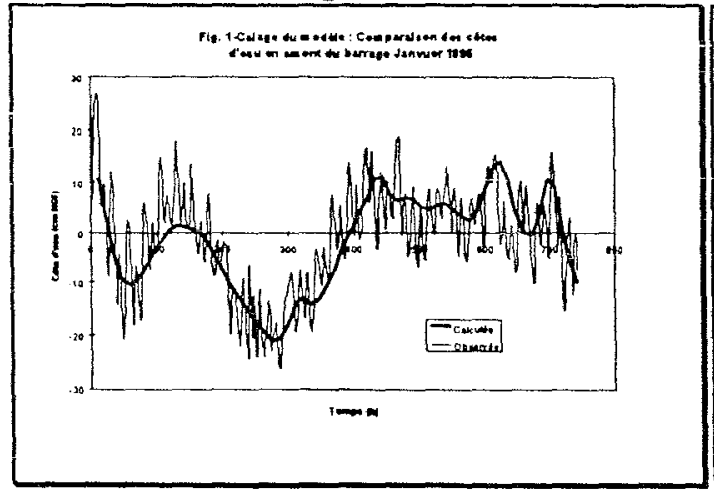

Figure 2

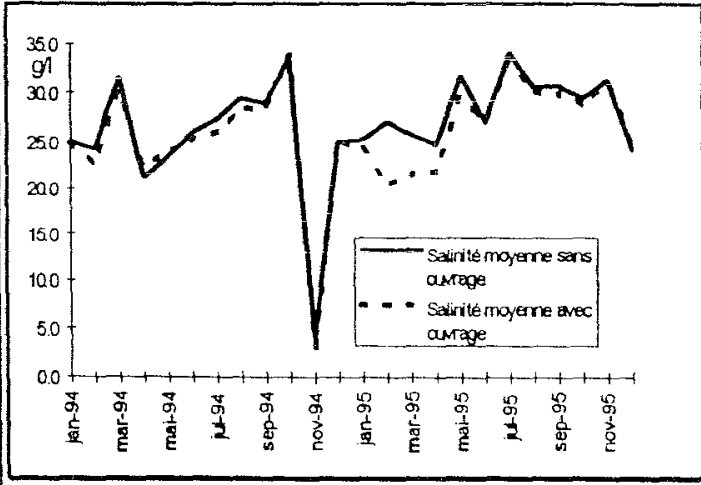


Carte 2 Champ de vitesses
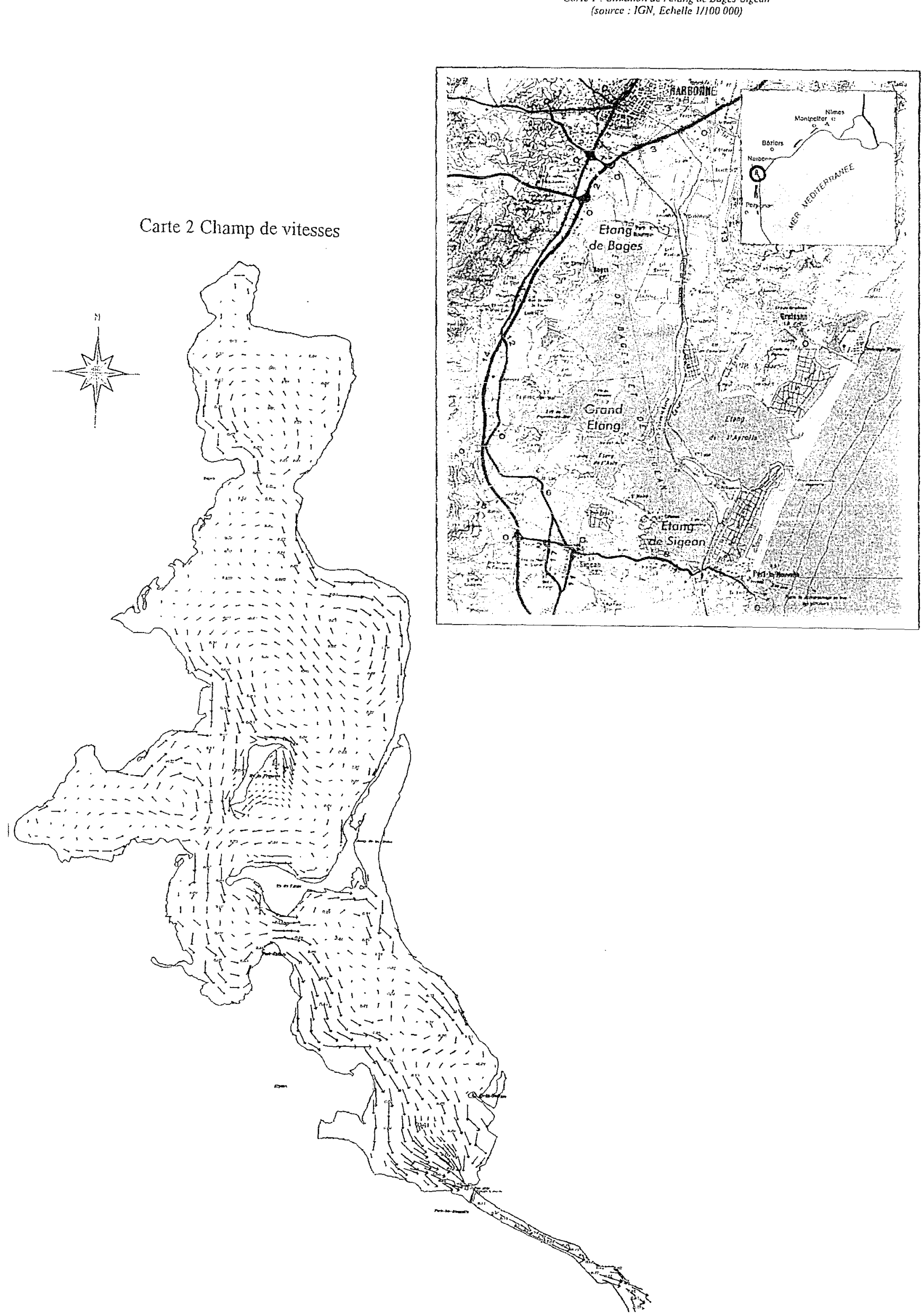